\title{
Attitudes toward long-acting injectable antipsychotics among patients with schizophrenia in Japan
}

\author{
Norio Sugawara ${ }^{1,2}$ \\ Shuhei Kudo² \\ Masamichi Ishioka ${ }^{3}$ \\ Yasushi Sato 2,4 \\ Kazutoshi Kubo ${ }^{3}$ \\ Norio Yasui-Furukori ${ }^{2}$ \\ 'Department of Clinical Epidemiology, \\ Translational Medical Center, National \\ Center of Neurology and Psychiatry, \\ Kodaira, Japan; ${ }^{2}$ Department of \\ Neuropsychiatry, Hirosaki University \\ School of Medicine, Hirosaki, Japan; \\ ${ }^{3}$ Department of Psychiatry, Hirosaki- \\ Aiseikai Hospital, Hirosaki, Japan; \\ ${ }^{4}$ Department of Psychiatry, Seihoku- \\ Chuoh Hospital, Goshogawara, Japan
}

Correspondence: Norio Sugawara Department of Clinical Epidemiology, Translational Medical Center, National Center of Neurology and Psychiatry, 4-I-I Ogawa-Higashi, Kodaira City,

Tokyo, I87-855I, Japan

$\mathrm{Tel}+8|4234| 27 \mid 2$

Fax +8I 423463503

Email nsuga3@yahoo.co.jp
This article was published in the following Dove Medical Press journal: Neuropsychiatric Disease and Treatment

Background: Long-acting injectable antipsychotics (LAIs) are regarded as an important alternative to oral medication for patients with schizophrenia. However, LAIs remain underutilized in clinical practice.

Aims: The aims of this investigation were to 1) obtain information on patients' attitudes toward LAIs and 2) assess factors associated with patients' acceptance of LAIs, and 3 ) identify predictors of the discrepancy between patients and referring psychiatrists' opinions regarding the appropriateness for LAIs.

Methods: Anonymized data were collected from a questionnaire distributed to 159 patients with schizophrenia and their referring psychiatrists at three psychiatric hospitals between February 2014 and July 2014. The patients completed an original questionnaire developed to evaluate their attitudes regarding LAIs. Regarding the appropriateness of LAI prescription, patients and their referring psychiatrists were asked to rate, on a 5-point scale, how appropriate they felt the depot prescription was for the patients. The participants also answered instruments to assess symptom severity, antipsychotic-induced extrapyramidal symptoms, functions, quality of life, and self-esteem levels.

Results: Patients currently on LAIs have favorable attitudes toward LAIs with respect to side effects, relapse prevention, efficacy, pain, and cost. Expectation of relapse prevention was significantly associated with patients' acceptance of LAIs (answering that those drugs are appropriate for their own treatment). In addition, the discrepancy between the patients' and referring psychiatrists' opinions regarding the appropriateness of LAI treatment was significantly associated with symptom severity, expectation of relapse prevention, belief that LAIs are painful, and belief that LAIs offer a reduced range of antipsychotic choices.

Conclusion: Attitudes toward LAIs need to be considered when deciding whether to prescribe this formulation. Access to information on LAIs, including their benefit in relapse prevention, might enhance the acceptance and use of this formulation among patients with schizophrenia. Keywords: adherence, long-acting injectable antipsychotics, schizophrenia, attitude, Japanese

\section{Introduction}

Maintenance with antipsychotic medications has long been known as a critical modality of treatment for patients with schizophrenia. However, approximately $40 \%-50 \%$ of patients are estimated to have poor adherence to their treatment regimen. ${ }^{1}$ Because poor adherence is associated with a high risk for relapse as well as functional outcomes, ${ }^{2-4}$ improving adherence is an important challenge in treating patients with schizophrenia. In this context, long-acting (depot) injectable antipsychotics (LAIs) are regarded as an important alternative for treating patients with poor adherence, although such 
treatments have shortcomings including a limited number of medications with this formulation, reduced flexibility in dose adjustment, and delayed disappearance of adverse effects.

Although some large randomized controlled trials (RCTs) have not shown significant difference in efficacy between LAIs and oral antipsychotics, ${ }^{5,6}$ the experimental nature of RCTs, such as enhanced compliance and selective recruitment, might explain these results. In naturalistic settings, several studies have demonstrated the superior effectiveness of LAIs over oral antipsychotics in improving quality of life, reducing hospitalization rates and treatment discontinuation; ${ }^{7,8}$ however, LAIs remain underutilized in clinical practice. ${ }^{9,10}$ To date, patients' and psychiatrists' attitudes toward LAIs that may affect clinical practice have been investigated. ${ }^{11,12}$ Although psychiatrists recognize the advantage of LAIs, they mostly recommend changing to an LAI formulation when patients practice poor adherence or request LAI treatment. ${ }^{11-13}$ On the other hand, psychiatric patients have a more negative attitude toward LAI treatment than psychiatrists. ${ }^{11,12}$ Among psychiatric patients, LAIs were regarded as stigmatic and were believed to constrict their freedom and cause more adverse effects than oral medications..$^{11,12,14}$ The abovementioned negative attitudes of psychiatric patients might affect their self-esteem as LAI users. However, few studies have investigated factors predicting discrepancies in perception of LAI treatment between patients and psychiatrists.

Furthermore, previous studies have indicated that the use of LAIs varies between countries. ${ }^{15-18}$ In Japan, psychiatrists reported that they had little experience with LAIs, ${ }^{13}$ which might lead to underutilization of this formulation. ${ }^{9}$ Differences in psychiatrists' or patients' attitudes as well as health care systems might affect this discrepancy between countries. However, there has been limited evidence concerning attitudes toward LAIs among patients with schizophrenia in Japan.

The objectives of this investigation were to 1) obtain information on patients' attitudes toward LAIs and 2) assess factors associated with patients' acceptance of LAIs, and 3) identify predictors of the discrepancy between patients' and psychiatrists' opinions of the appropriateness of LAIs. To the best of our knowledge, this article presents the first study on the association between patients' acceptance of and attitudes toward LAIs in Japan.

\section{Methods}

\section{Participants}

This cross-sectional study comprised 159 outpatients (69 males and 90 females) with either schizophrenia or schizoaffective disorder according to the $D S M-I V$, who were treated at three psychiatric hospitals in Japan between February 2014 and July 2014. The research protocol was approved by the Independent Ethics Committee of the Hirosaki University School of Medicine (approval number 2014-6). The capacity of the patients to provide informed consent was assessed by their treating psychiatrists. Written informed consent was obtained from all study participants. A surrogate consent procedure was not employed in this study. This protocol was conducted in accordance with the Declaration of Helsinki. The medical records of the patients were reviewed for obtaining data of the diagnoses, medical histories, and demographic data (age, sex, and duration of education). To obtain comparable medication values for each patient, we converted each medication to chlorpromazine (CP) equivalents. ${ }^{19}$

\section{Assessment}

We employed the Clinical Global Impressions-Severity of Illness scale (CGI-S) to assess symptom severity. This is a widely used rating tool applicable to all psychiatric disorders including schizophrenia and practicing psychiatrists can easily evaluate their patients. Based on all available information, including patient's history, psychiatric symptoms, psychosocial circumstances, and behavior, psychiatrists rate the participants on a 7-point scale from 1 (normal, not at all ill) to 7 (among the most extremely ill patients). ${ }^{20}$

Extrapyramidal symptoms caused by antipsychotic drugs were evaluated with the Drug-Induced Extrapyramidal Symptom Scale (DIEPSS) based on objective observations. ${ }^{21}$ It consists of nine items (gait, bradykinesia, sialorrhea, rigidity, tremor, akathisia, dystonia, dyskinesia, and overall severity), each rated on a 5-point scale from 0 (normal) to 4 (severe). In this study, we used a sum of nine items as total DIEPSS scores.

The Global Assessment of Functioning (GAF) is a rating scale to evaluate a symptom severity or the level of functioning. In case that evaluation of symptom and functioning is discordant, clinicians are directed to use the rating which is more severe. Scores range from 0 to 100 where higher scores indicate less severe symptoms or function at a higher level. ${ }^{22}$

To survey health-related quality of life (QOL), the 36-item Short Form Health Survey version 2 (SF-36v2 ${ }^{\circledR}$ ) was conducted on our participants. ${ }^{23,24}$ The SF-36v $2^{\circledR}$ is a 36 -item, self-administered questionnaire, which has been standardized to have a mean of 50 and a standard deviation (SD) of 10. In this study, we used two summary measures: the Physical Composite Summary and the Mental Composite Summary. 
To evaluate the self-esteem levels, we employed the Rosenberg Self-Esteem Scale, which has high reliability and validity. ${ }^{25}$ It consists of ten items, in which five have positively worded statements (positive self-esteem) and five have negatively worded ones (negative self-esteem). The items are answered on a 5-point scale ranging from 1 (strongly disagree) to 4 (strongly agree). The scoring of five negative items were reversed with higher scores indicating more positive perceptions of self-esteem.

After reviewing earlier investigations with comparable objectives, ${ }^{11,26,27}$ we constructed a brief questionnaire (Q1-Q8) concerning attitudes toward LAIs. The questions in the section were to be answered by stating "yes" or "no" only. The detailed questions are summarized in Table 1 . Regarding their current treatment satisfaction, patients rated on a 5 -point scale ( $1=$ very satisfied, $2=$ satisfied, $3=$ neutral, 4=dissatisfied, $5=$ very dissatisfied). Regarding acceptance or appropriateness of LAI prescription, patients and their referring psychiatrists were asked to answer on a 5-point scale $(1=$ very appropriate, $2=$ appropriate, $3=$ neutral, $4=$ not appropriate, $5=$ not appropriate at all), how appropriate they felt the depot prescription was for the patients. Those answering "1 or 2" were grouped as answering "appropriate," while those who answered " 4 or 5" were considered as answering "not appropriate." Furthermore, those who were assessed as appropriate by oneself and as not appropriate by one's referring psychiatrists were classified as type A discrepancy group, whereas those who were assessed as not appropriate by oneself and as appropriate by one's referring psychiatrists were classified as type B discrepancy group (Figure 1).

\section{Statistical analyses}

Student's unpaired $t$-test for continuous variables or chisquared test for categorical variables was used to evaluate the difference in the main characteristics and attitudes between patients on LAIs and those on oral antipsychotics. Regarding their current treatment satisfaction, a statement was a priori defined as being satisfied if the mean rating was below the numeric center of the scale ( $3=$ =neutral rating). We employed a one-sample $t$-test to test whether ratings significantly differed from the numeric center of the scale. The data are presented as mean and SD.

After controlling the effect of covariates (age, gender, amount of education, duration of illness, CGI-S, GAF, and DIEPSS score), multivariate logistic regression analysis with a forward selection method was performed to assess the influence of attitude (Q1-Q8) toward LAIs as a predictor of patients' acceptance of LAIs (answering that those are appropriate for oneself) among those treated with oral antipsychotics only. We selected covariates of this model based on previous findings. ${ }^{28,29}$ Under the same conditions, we also conducted an analysis to assess the influence of attitude toward LAIs as a predictor of type A and B discrepancy regarding appropriateness of LAI treatment among patients treated with oral antipsychotics only. The level of statistical significance is set at 0.05 and analyses were conducted using SPSS Statistics PC software for Windows, Version 24 (IBM Corporation, Armonk, NY, USA).

\section{Results}

Twenty-nine (18.2\%) participants were on LAIs, and 130 $(81.8 \%)$ were on oral antipsychotics, of whom eight were previously on LAIs. Participants on LAIs were significantly older and had a longer duration of illness and higher total $\mathrm{CP}$ equivalent dose than those who were taking oral antipsychotics. No other group differences were found (Table 2). There was no significant difference in current treatment satisfaction between patients on LAIs and those on oral

Table I Patient attitudes toward LAls according to the current formulation among patients with schizophrenia in Japan

\begin{tabular}{|c|c|c|c|c|c|c|}
\hline & \multirow[b]{2}{*}{ Do you believe... } & \multicolumn{2}{|c|}{$\begin{array}{c}\text { Oral } \\
\text { antipsychotics }\end{array}$} & \multicolumn{2}{|c|}{ LAls } & \multirow{2}{*}{$\begin{array}{c}\chi^{2} \\
\text { P-value }\end{array}$} \\
\hline & & Yes & & Yes & & \\
\hline QI & ... that depot have milder side effects than oral treatment? & $18.0 \%$ & $(23 / 128)$ & $76.0 \%$ & $(19 / 25)$ & $<0.001$ \\
\hline Q2 & ... that by depot you are better protected against relapse? & $32.8 \%$ & $(42 / 128)$ & $79.2 \%$ & $(19 / 24)$ & $<0.001$ \\
\hline Q3 & ... that depot offer better efficacy than oral treatment? & $43.0 \%$ & $(55 / 128)$ & $79.2 \%$ & $(19 / 24)$ & 0.001 \\
\hline Q4 & ... that depot limit the autonomy of the patient? & $38.6 \%$ & $(49 / 127)$ & $28.0 \%$ & $(7 / 25)$ & 0.316 \\
\hline Q5 & ... that depot are very painful? & $58.6 \%$ & $(75 / 128)$ & $29.2 \%$ & $(7 / 24)$ & 0.008 \\
\hline Q6 & ... that antipsychotic doses are lower with depot treatment than with oral drugs? & $51.6 \%$ & $(66 / 128)$ & $72.0 \%$ & $(18 / 25)$ & 0.060 \\
\hline Q7 & ... that depot are more expensive than oral treatment? & $66.4 \%$ & $(85 / 128)$ & $32.0 \%$ & $(8 / 25)$ & 0.001 \\
\hline Q8 & ... that depot offer a reduced range of antipsychotic choices? & $57.8 \%$ & $(74 / 128)$ & $70.8 \%$ & $(17 / 24)$ & 0.232 \\
\hline
\end{tabular}

Abbreviation: LAls, long-acting injectable antipsychotics. 


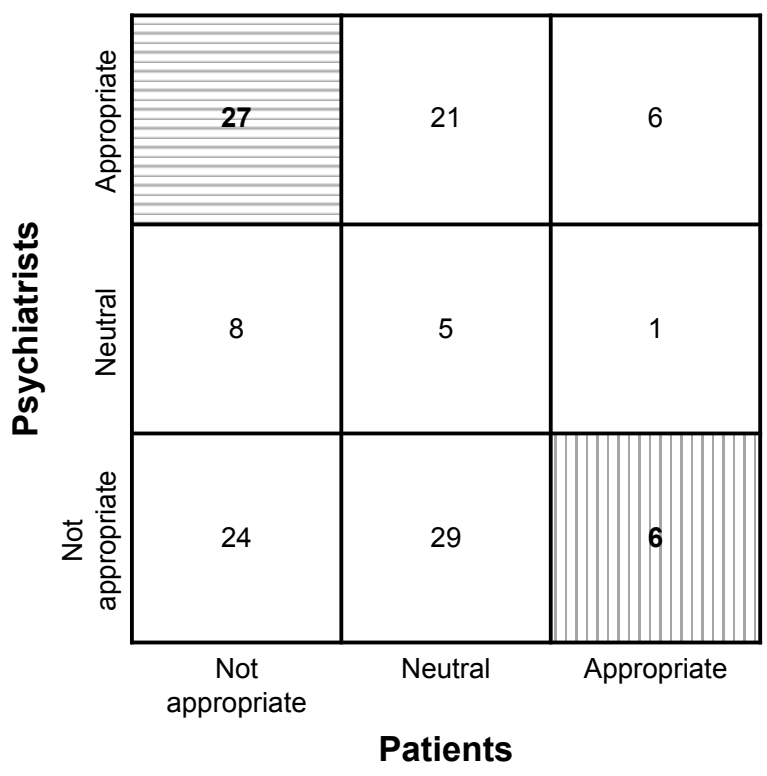

Figure I Responses of patients and their psychiatrists regarding the appropriateness of LAl treatment among patients taking oral antipsychotic medication only. Notes: The box shaded with vertical lines represents type A discrepancy (patients evaluated the appropriateness of LAI treatment higher than their referring psychiatrists). On the other hand, the box shaded with horizontal lines represents type B discrepancy (patients evaluated the appropriateness of LAI treatment lower than their referring psychiatrists).

Abbreviation: LAI, long-acting injectable antipsychotic.

antipsychotics ( $P=0.289)$. Ratings below the numeric center of the scale (indicating satisfaction with current treatment) were found for patient satisfaction with both LAIs and oral antipsychotics $(2.0 \pm 0.8, P<0.001$, and $2.2 \pm 0.8, P<0.001$, respectively).
Table 1 shows attitudes toward LAIs according to the current formulation among patients with schizophrenia in Japan. Patients currently on LAIs have favorable attitudes concerning side effects (Q1), relapse prevention (Q2), efficacy (Q3), pain (Q5), and cost (Q7) toward LAIs.

To assess the influence of attitudes (Q1-Q8) toward LAIs as a predictor of patients' acceptance of LAIs (answering that those drugs would be appropriate for them) among those treated with oral antipsychotics only, we performed a multivariate logistic regression analysis with a forward selection method. Patients' response to Q2 (expectation of relapse prevention) was significantly associated with answering that LAI treatment would be appropriate for them (Table 3).

Table 4 shows the factors associated with doctor-patient discrepancy regarding the appropriateness of LAIs among patients treated with oral antipsychotics only. Regarding type A discrepancy, responses to Q5 (belief that LAIs are painful) approached statistical significance $(P=0.06)$. Responses to Q2, Q5, and Q8 (belief that LAIs offer a reduced range of antipsychotic choices) were significantly associated with type B discrepancy.

\section{Discussion}

The advantages and disadvantages of LAIs in clinical practice are still controversial, and patients with LAIs indication are indicated and are not necessarily prescribed this formulation. A better understanding of attitudes toward LAIs would likely improve their acceptance and use in

Table 2 Clinical and demographic characteristics according to current formulation

\begin{tabular}{|c|c|c|c|}
\hline & Oral antipsychotics & LAls & t-test \\
\hline & $($ mean \pm SD) & $($ mean \pm SD) & $P$-value \\
\hline Age (years) & $43.3 \pm 13.4$ & $49.1 \pm 11.9$ & 0.034 \\
\hline Duration of illness (years) & $16.1 \pm 10.1$ & $22.1 \pm 8.4$ & 0.004 \\
\hline Duration of education (years) & $11.9 \pm 2.1$ & $11.5 \pm 1.5$ & 0.337 \\
\hline CGI-S & $4.0 \pm 1.1$ & $4.4 \pm 0.9$ & 0.137 \\
\hline DIEPSS total score & $2.4 \pm 3.9$ & $4.3 \pm 3.8$ & 0.095 \\
\hline GAF & $57.7 \pm 14.6$ & $53.6 \pm 12.2$ & 0.129 \\
\hline Total CP equivalent dose (mg) & $504 \pm 376$ & $77 I \pm 478$ & 0.008 \\
\hline $\mathrm{CP}$ equivalent dose of oral administration (mg) & $504 \pm 376$ & $505 \pm 488$ & 0.995 \\
\hline \multicolumn{4}{|l|}{ SF-36v $2^{\circledR}$ score } \\
\hline Physical component summary & $39.8 \pm 16.1$ & $35.1 \pm 22.7$ & 0.446 \\
\hline Mental component summary & $45.0 \pm 10.9$ & $48.5 \pm 13.8$ & 0.272 \\
\hline \multicolumn{4}{|l|}{ Rosenberg Self-Esteem Scale } \\
\hline Positive self-esteem & $12.4 \pm 3.8$ & $13.0 \pm 3.7$ & 0.509 \\
\hline \multirow[t]{2}{*}{ Negative self-esteem } & $11.8 \pm 3.6$ & $11.4 \pm 3.7$ & 0.640 \\
\hline & $\%$ & $\%$ & $x^{2}$ \\
\hline Gender (proportion of males) & $43.1(56 / 130)$ & $44.8(13 / 29)$ & 0.863 \\
\hline
\end{tabular}

Abbreviations: CGI-S, Clinical Global Impressions-Severity of Illness scale; CP, chlorpromazine; DIEPSS, Drug-Induced Extrapyramidal Symptoms Scale; GAF, Global Assessment of Functioning; LAls, long-acting injectable antipsychotics; SF-36v2 ${ }^{\circledR}, 36$-Item Short-Form Health Survey version 2. 
Table 3 Factors associated with acceptance of LAls (response that these drugs are appropriate for one's own case) among patients treated with oral antipsychotics only

\begin{tabular}{llllll}
\hline & B & Standard error & Wald value & $P$-value & Odds ratio \\
\hline Q2 & I.36 & 0.61 & 4.91 & 0.027 & $3.89(1.17-12.91)$ \\
\hline
\end{tabular}

Notes: After adjusting for confounding factors (age, gender, amount of education, duration of illness, CGI-S score, GAF score, and DIEPSS score), we performed a multivariate logistic regression analysis with a forward selection method to assess the influence of attitudes on acceptance of LAls among patients treated with oral antipsychotics only. Abbreviations: CGI-S, Clinical Global Impressions-Severity of Illness scale; DIEPSS, Drug-Induced Extrapyramidal Symptoms Scale; GAF, Global Assessment of Functioning; LAls, long-acting injectable antipsychotics.

patients with schizophrenia. The objective of this study was to assess the attitudes of schizophrenia patients toward LAIs. In our survey, both patients who were currently on LAIs and those who took oral antipsychotics were satisfied with their current treatment. Furthermore, patients currently on LAIs had favorable attitudes toward LAIs with respect to side effects (Q1), relapse prevention (Q2), efficacy (Q3), pain (Q5), and cost (Q7). Response to Q2 (expectation of relapse prevention) was significantly associated with patients' acceptance of LAIs (answering that those drugs would be appropriate for them). Expectation of relapse prevention (Q2), belief that LAIs are painful (Q5), and belief that LAIs limit the range of antipsychotic options (Q8) were significantly associated with type B discrepancy (patients evaluate the appropriateness of LAI treatment lower than their referring psychiatrists).

Previous studies have shown that patients' preference for LAIs as favorable antipsychotic treatment depends on their experience with the formulation. A study from German reported that only $23 \%$ of patients who had never taken LAIs considered this mode of application acceptable, compared with $45 \%$ of patients previously on LAIs and $73 \%$ of the patients currently on LAIs. ${ }^{26}$ Another study from Australia demonstrated that more than half of patients receiving LAI treatment rated their current medication helpful, even among patients lacking insight. ${ }^{30}$ Regarding relapse prevention, lowering the total antipsychotic dose, and controlling psychiatric symptoms, more than half of patients currently on LAIs showed positive attitudes toward LAI treatment. ${ }^{12,26}$

Although our results indicated that more than half of patients did not regard LAI treatment as limiting their autonomy, ethical concerns regarding coercion have been raised historically. ${ }^{31}$ A previous study showed that patients, more than psychiatrists, felt that LAIs restricted patient autonomy. ${ }^{11}$ In addition, patients currently on LAIs felt that their current treatment was more coercive than patients currently on oral antipsychotics. Specifically, more participants on LAIs felt that people tried to force them to take medication. ${ }^{14}$ This discrepancy between our results and previous findings may be related to differences in the nature of the scaling method (binary or Likert response), ethnocultural factors, or history of involuntary hospitalizations among study participants. Regardless of the abovementioned discrepancy, psychiatrists should minimize patients' feelings of coercion in the shared decision-making process for commencing LAI treatment.

Previous findings concerning underuse and poor provision of information concerning LAIs may indicate a

Table 4 Factors associated with doctor-patient discrepancy regarding the appropriateness of long-acting injectable antipsychotics treatment among patients treated with oral antipsychotics only

\begin{tabular}{llllll}
\hline & B & Standard error & Wald value & P-value & Odds ratio \\
\hline $\begin{array}{l}\text { Type A discrepancy } \\
\text { Q5 }\end{array}$ & -2.09 & 1.11 & 3.52 & 0.06 & $0.12(0.01-1.10)$ \\
$\begin{array}{l}\text { Type B discrepancy } \\
\text { CGI-S }\end{array}$ & 0.60 & 0.22 & & & \\
Q2 & -1.79 & 0.73 & 7.22 & 0.01 & $1.82(1.18-2.82)$ \\
Q5 & 1.63 & 0.65 & 5.96 & 0.01 & $0.17(0.04-0.70)$ \\
Q8 & -1.25 & 0.59 & 6.25 & 0.01 & $5.10(1.42-18.31)$ \\
\hline
\end{tabular}

Notes: After adjusting for confounding factors (age, gender, amount of education, duration of illness, CGI-S score, GAF score, and DIEPSS score), we performed a multivariate logistic regression analysis with a forward selection method to assess the influence of attitudes (Q I-Q8) on discrepancy between patients' and psychiatrists' responses on the appropriateness of LAls treatment. Type A discrepancy: patients evaluated the appropriateness of LAI treatment higher than their referring psychiatrists. Type B discrepancy: patients evaluated the appropriateness of LAI treatment lower than their referring psychiatrists.

Abbreviations: CGI-S, Clinical Global Impressions-Severity of Illness scale; DIEPSS, Drug-Induced Extrapyramidal Symptoms Scale; GAF, Global Assessment of Functioning; LAls, long-acting injectable antipsychotics. 
potential need for LAIs among patients currently receiving oral antipsychotic treatment. ${ }^{11}$ Although satisfaction with oral antipsychotics and fear of side effects, needles, or pain were raised by patients with schizophrenia as reasons to refuse LAI treatment, ${ }^{32}$ the practice of offering information on the benefit of LAIs for relapse prevention might enhance prescription among patients with schizophrenia. On the other hand, patients with severe psychiatric symptoms or fear of pain might refuse LAI treatment if psychiatrists offered this formulation as an appropriate treatment option. Our results also indicate that the practice of offering information, including the benefit of LAIs for relapse prevention or the range of antipsychotics available in that formulation, might reduce discrepancies between patient and psychiatrist opinions on LAIs. Although we found several differences in attitudes toward LAI treatments between patients on oral antipsychotics and those on LAIs, there was no significant difference in current treatment satisfaction between groups. Comparison of health-related QOL or self-esteem also supports the results on patient satisfaction. Some ethical concerns regarding LAIs have been discussed. However, this formulation was at least satisfactory to patients currently using it.

Although more than half of participants answered that the antipsychotic dose would be lower for LAI treatment than for oral medication, the total CP equivalent dose was higher in patients currently on LAIs than in those on oral antipsychotic treatment. Despite their use of injectable medication, patients currently on LAIs received the same dose of oral antipsychotics as patients on oral antipsychotic treatment only. Although our participants currently on LAIs answered that this formulation had fewer side effects than oral treatment, their total DIEPSS scores tended to be higher than those of patients on oral treatment only. Since high doses of antipsychotics have been reported to cause extrapyramidal side effects, ${ }^{33}$ psychiatrists should refrain from coadministering oral antipsychotics and LAIs.

\section{Limitations}

Several limitations of this study should be acknowledged. First, our study is limited by the fact that patients were merely asked hypothetically about the appropriateness of LAI prescription. Participant responses may not accurately reflect whether they would actually accept the prescription of LAIs in a clinical setting. Second, several potential confounding factors, such as employment status, capacity to consent, insight into illness, and history of involuntary hospitalization, all of which may contribute to patient assessments of the appropriateness of LAI treatment, were not assessed in this study. Specifically, we could not obtain information concerning whether patients currently taking LAIs felt coerced into taking this type of formulation. Third, the severity of psychiatric symptoms was assessed by the CGI-S, rather than by a clinician-administered structured interview. Although the CGI-S correlates well with other rating scales, such as the PANSS, ${ }^{34}$ the former does not measure all the important dimensions of schizophrenia symptoms. Fourth, the participants currently taking LAIs were older and had a longer duration of illness than those on oral antipsychotics. Previous studies have shown that psychiatrists also hesitate to prescribe LAIs for first-episode schizophrenia. ${ }^{13,26,35}$ Their presumption that this formulation is mostly appropriate for chronic patients might affect the characteristics of our participants. However, differences in age and illness duration could affect patients' attitudes toward LAIs. Fifth, our participants may not be representative of all Japanese patients with schizophrenia because our study was conducted using a small sample size from a rural district only. The abovementioned limitations should be addressed in future studies.

\section{Conclusion}

Attitudes toward LAIs need to be considered when deciding whether to prescribe this formulation. Providing information about the properties of LAIs, including relapse prevention, might enhance the acceptance and use of this formulation among patients with schizophrenia. Psychiatrists should minimize patients' feelings of coercion in the shared decision-making process of commencing LAI treatment.

\section{Acknowledgments}

We are grateful to all the volunteers who participated in this study. Funding for this study was provided by the Hirosaki Research Institute for the Neurosciences. The funder had no role in the study design, data collection, data analysis or writing of the report. Abstracts presented at the 30th CINP World Congress of Neuropsychopharmacology, Seoul, are available online (https://academic.oup.com/ijnp/article/19/ Suppl 1/45/2946071).

\section{Disclosure}

The authors report no conflicts of interest in this work.

\section{References}

1. Lacro JP, Dunn LB, Dolder CR, Leckband SG, Jeste DV. Prevalence of and risk factors for medication nonadherence in patients with schizophrenia: a comprehensive review of recent literature. J Clin Psychiatry. 2002;63(10):892-909.

2. Ascher-Svanum H, Faries DE, Zhu B, Ernst FR, Swartz MS, Swanson JW. Medication adherence and long-term functional outcomes in the treatment of schizophrenia in usual care. J Clin Psychiatry. 2006;67(3):453-460. 
3. Novick D, Haro JM, Suarez D, Perez V, Dittmann RW, Haddad PM. Predictors and clinical consequences of non-adherence with antipsychotic medication in the outpatient treatment of schizophrenia. Psychiatry Res. 2010;176(2-3):109-113.

4. Alvarez-Jimenez M, Priede A, Hetrick SE, et al. Risk factors for relapse following treatment for first episode psychosis: a systematic review and meta-analysis of longitudinal studies. Schizophr Res. 2012; 139(1-3):116-128

5. Macfadden W, Ma YW, Thomas Haskins J, Bossie CA, Alphs L. A prospective study comparing the long-term effectiveness of injectable risperidone long-acting therapy and oral aripiprazole in patients with schizophrenia. Psychiatry. 2010;7(11):23-31.

6. Rosenheck RA, Krystal JH, Lew R, et al. Long-acting risperidone and oral antipsychotics in unstable schizophrenia. N Engl J Med. 2011; 364(9):842-851.

7. Pietrini F, D'Anna G, Tatini L, et al. Changes in attitude towards LAI antipsychotic maintenance treatment: a two-year follow-up study. Eur Psychiatry. 2018;53:58-65.

8. Kishimoto T, Hagi K, Nitta M, et al. Effectiveness of long-acting injectable vs oral antipsychotics in patients with schizophrenia: a metaanalysis of prospective and retrospective cohort studies. Schizophr Bull. 2018;44(3):603-619.

9. Cheung S, Hamuro Y, Mahlich J, Nakahara T, Sruamsiri R, Tsukazawa S. Drug utilization of Japanese patients diagnosed with schizophrenia an administrative database analysis. Clin Drug Investig. 2017;37(6): 559-569.

10. Kishimoto T, Sanghani S, Russ MJ, et al. Indications for and use of long-acting injectable antipsychotics: consideration from an inpatient setting. Int Clin Psychopharmacol. 2017;32(3):161-168.

11. Jaeger M, Rossler W. Attitudes towards long-acting depot antipsychotics: a survey of patients, relatives and psychiatrists. Psychiatry Res. 2010; 175(1-2):58-62.

12. Tsang HW, Fong MW, Fung KM, Chung RC. Perceptions on efficacy and side effects of conventional depot antipsychotics (CDA) and atypical depot antipsychotics (ADA): psychiatrists versus patients in Hong Kong. Int J Psychiatry Clin Pract. 2010;14(1):23-32.

13. Fujii Y, Iwata N, Takahashi K. Large-scale about psychiatrists' use, knowledge and attitudes to depot antipsychotics: a comparison between Japanese and German psychiatrist. Jpn J Clin Psychpharmacol. 2012; $15: 797-810$

14. Patel MX, de Zoysa N, Bernadt M, Bindman J, David AS. Are depot antipsychotics more coercive than tablets? The patient's perspective. J Psychopharmacol. 2010;24(10):1483-1489.

15. Fourrier A, Gasquet I, Allicar MP, Bouhassira M, Lépine JP, Bégaud B. Patterns of neuroleptic drug prescription: a national cross-sectional survey of a random sample of French psychiatrists. Br J Clin Pharmacol. 2000;49(1):80-86.

16. Barnes TR, Shingleton-Smith A, Paton C. Antipsychotic long-acting injections: prescribing practice in the UK. Br J Psychiatry Suppl. 2009; $52: \mathrm{s} 37-\mathrm{s} 42$.

17. Williams R, Kopala L, Malla A, Smith G, Love L, Balshaw R. Medication decisions and clinical outcomes in the Canadian national outcomes measurement study in schizophrenia. Acta Psychiatrica Scandinavica. 2006;113(s430):12-21.
18. West JC, Marcus SC, Wilk J, Countis LM, Regier DA, Olfson M. Use of depot antipsychotic medications for medication nonadherence in schizophrenia. Schizophr Bull. 2008;34(5):995-1001.

19. Inada T, Inagaki A. Psychotropic dose equivalence in Japan. Psychiatry Clin Neurosci. 2015;69(8):440-447.

20. Busner J, Targum SD. The clinical global impressions scale: applying a research tool in clinical practice. Psychiatry. 2007;4(7):28-37.

21. Inada T. Evaluation and diagnosis of drug-induced extrapyramidal symptoms. In: Yagi G, editor. Commentary on the DIEPSS and Guide to Its Usage. Tokyo: Seiwa Publishers; 1996;11-60.

22. Aas IH. Guidelines for rating global assessment of functioning (GAF). Ann Gen Psychiatry. 2011;10(1):2.

23. Ware JE, Sherbourne CD. The MOS 36-item short-form health survey (SF-36). I. conceptual framework and item selection. Med Care. 1992;30(6):473-483.

24. Taft C, Karlsson J, Sullivan M. Do SF-36 summary component scores accurately summarize subscale scores? Qual Life Res. 2001;10(5): 395-404.

25. Rosenberg M. Society and the Adolescent Self-Image. Princeton University Press: Princeton; 1965.

26. Heres S, Schmitz FS, Leucht S, Pajonk FG. The attitude of patients towards antipsychotic depot treatment. Int Clin Psychopharmacol. 2007;22(5):275-282.

27. Kim SW, Lee YH, Jang JE, et al. Comparison of attitudes toward long-acting injectable antipsychotics among psychiatrists and patients. Int Clin Psychopharmacol. 2013;28(2):80-86.

28. Patel MX, de Zoysa N, Bernadt M, David AS. A cross-sectional study of patients' perspectives on adherence to antipsychotic medication: depot versus oral. J Clin Psychiatry. 2008;69(10):1548-1556.

29. Patel MX, de Zoysa N, Bernadt M, David A. Depot and oral antipsychotics: patient preferences and attitudes are not the same thing. J Psychopharmacol. 2009;23(7):789-796.

30. Castle D, Morgan V, Jablensky A. Antipsychotic use in Australia: the patients' perspective. Aust N Z J Psychiatry. 2002;36(5):633-641.

31. Roberts LW, Geppert CM. Ethical use of long-acting medications in the treatment of severe and persistent mental illnesses. Compr Psychiatry. 2004;45(3):161-167.

32. Iyo M, Nakagome K, Wakamatsu A, Takahashi K. Fact-finding survey on awareness and utilization of depot antipsychotics for schizophreniaresults of questionnaire survey of psychiatric nurses and schizophrenia patients in terms of relapse prevention and medication. Jap J Psychiatry. 2014;19(1):55-62.

33. Divac N, Prostran M, Jakovcevski I, Cerovac N. Second-generation antipsychotics and extrapyramidal adverse effects. Biomed Res Int. 2014;2014(9):1-6.

34. Leucht S, Kane JM, Kissling W, Hamann J, Etschel E, Engel RR. What does the PANSS mean? Schizophr Res. 2005;79(2-3):231-238.

35. Kirschner M, Theodoridou A, Fusar-Poli P, Kaiser S, Jäger M. Patients' and clinicians' attitude towards long-acting depot antipsychotics in subjects with a first episode of psychosis. Ther Adv Psychopharmacol. 2013;3(2):89-99.
Neuropsychiatric Disease and Treatment

\section{Publish your work in this journal}

Neuropsychiatric Disease and Treatment is an international, peerreviewed journal of clinical therapeutics and pharmacology focusing on concise rapid reporting of clinical or pre-clinical studies on a range of neuropsychiatric and neurological disorders. This journa is indexed on PubMed Central, the 'PsycINFO' database and CAS,

\section{Dovepress}

and is the official journal of The International Neuropsychiatric Association (INA). The manuscript management system is completely online and includes a very quick and fair peer-review system, which is all easy to use. Visit http://www.dovepress.com/testimonials.php to read real quotes from published authors. 